\title{
MULTIPLE ODONTOGENIC KERATOCYSTS IN A NON-SYNDROMIC YOUNG PATIENT: AN UNUSUAL CASE REPORT
}

\author{
Ravish Mishra, ${ }^{1}$ Laxmi Kandel, ${ }^{1}$ Deepak Yadav, ${ }^{1}$ Shashank Tripathi, ${ }^{1}$ Bijay Karki, ${ }^{1}$ Nitesh Chaurasia, ${ }^{1}$ Sunil Kumar Singh ${ }^{1}$
}

\begin{abstract}
Odontogenic keratocyst (OKC) is a benign intraosseous lesion with invasive and aggressive behavior. It comprises approximately $2-21.8 \%$ of all jaw cysts. Odontogenic keratocysts (OKCs) are believed to arise from remnants of the dental lamina most common site in the molar ramus area. OKCs have a specific histopathologic appearance and are found to be locally aggressive and have a high recurrence rate, thus requires close long-term follow-up. OKCs are one component of the Gorlin-Goltz syndrome and all patients with multiple OKCs should be evaluated for this syndrome. In this paper, we present a case of a 13-year old non-syndromic female patient with multiple OKCs located at symphysis and bilateral mandibular angle region who was treated surgically with no obvious post-operative complications during follow-up period.
\end{abstract}

\section{KEYWORDS}

Intraosseous, Mandible, Odontogenic keratocyst.

1 Department of Oral and Maxillofacial Surgery, UCMS College of Dental Surgery, Bhairahawa, Nepal

DOI: http//doi.org/10.3126/jucms.v9i01.37990

For Correspondence

Dr. Ravish Mishra

Department of Oral and Maxillofacial Surgery

UCMS College of Dental Surgery

Bhairahawa, Nepal

Email: drravishmishra84@gmail.com 


\section{INTRODUCTION}

Odontogenic keratocyst $(\mathrm{OKC})$ was first described and termed by Philipsen in 1956. Earlier in 1926, it was called by other terms such as cholesteatoma by Haur 1926, and Kostecka 1929. The incidence of OKC ranges from infant to old age with slight male predilection and mandible being most common site of occurrence with specificity in molar-ramus area. The peak incidence is during the second to fourth decades of life. ${ }^{2}$ It comprises $2-21.8 \%$ of all jaw cysts. ${ }^{3}$

Previously OKC was classified under developmental odontogenic cyst of jaw by WHO in 1971 and 1992. WHO classification of odontogenic tumors in 2005 , it has been given the term keratocystic odontogenic tumor. ${ }^{4}$ However, in 2017, the new WHO classification of Head and Neck Pathology reback it into the cystic category. It is a benign uni- or multicystic intraosseous tumor of odontogenic origin. It is locally aggressive and infiltrative and has a distinctive lining of parakeratinized stratified squamous epithelium. It is reported that $5 \%$ of the patients with OKCs tend to have multiple OKCs. ${ }^{2}$ The occurrence of multiple OKCs is characteristically seen with cutaneous, skeletal, ocular and neurological abnormalities or syndrome associated. As OKCs are known for their recurrence, patients with multiple OKCs have a significantly higher recurrence rate of $30 \%$ compared to solitary keratocyst of $10 \%$. Reasons for recurrences of OKCs are: i) occurrence of satellite cysts, ii) presence of thin fragile lining. ${ }^{5}$

On the whole the incidence in children is relatively rare when compared to adults, of which sporadic OKC is dominant in incidence than multiple variants. This article presents an unusual case presentation of multiple OKCs in a nonsyndromic young patient.

\section{CASE REPORT}

A case of a 13-year female was referred from outside to the Department of Oral and Maxillofacial Surgery, UCMS College of Dental Surgery, Bhairahawa, Nepal with a chief complaint of swelling over submental region for two years and pus like discharge for two months. The swelling was small pea sized initially and increased gradually to the present size of approximately $3 \times 2 \mathrm{~cm}^{2}$. On extraoral examination, an erythematous swelling was present at submental region with obliteration of mento-labial sulcus (Figure 1). It was nonpainful, mildly tender and was associated with yellowish pus like discharge from it.

According to patient party, root canal treatment (RCT) was done for 31 and 41 about 1.5 years back at Government Hospital. Intraoral examination revealed swelling over lower labial vestibular region. Swelling extended from 33 to 43 which was firm and non-tender. A provisional diagnosis of submental space infection was made and patient was advised for orthopantomogram (OPG). OPG revealed multiple welldefined radiolucent lesions present at the symphysis region extending from 33 to 43, 38-region and 48-region (Figure 2). A probable diagnosis of infected radicular cyst was made in symphysis region and dentigerous cyst at 38 and 48 region of jaw. Routine blood investigation was done and found to be normal. Patient was advised to go for series of chest and skull radiographs to rule out Gorlin-Goltz syndrome but no abnormality was detected. Then incisional biopsy along with marsupialization done in the symphysis region (Figure 3) and extraction of tooth with complete cystic enucleation followed by primary closure in relation to 38 and 48 were done. Specimen were sent for histopathological examinations. Primary closure of the extra-oral wound was done. Histopathological examination revealed epithelium lined by stratified squamous epithelium which is para-keratinized having 4-6 layers of thickness in which basal cell layer is palisading arranged along with surface corrugation of epithelium and which was suggestive of OKCs in symphysis and both mandibular angle region (Figure 4). Hence, it was a case of multiple OKC of mandible which is not associated with any syndrome and was managed properly. The patient is on regular follow up for 8 months and till now there is no sign of recurrence during follow up OPG (Figure 5).

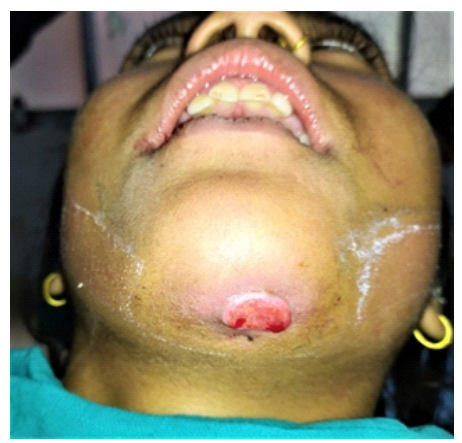

Figure 1. Pre-operative photograph of patients showing swelling over submental region with sinus drainage

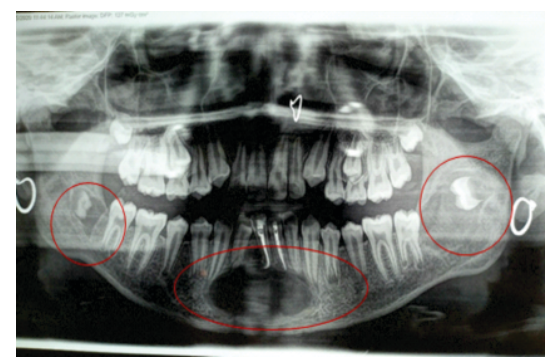

Figure 2. Pre-operative OPG showing multiple cystic lesions of mandible 


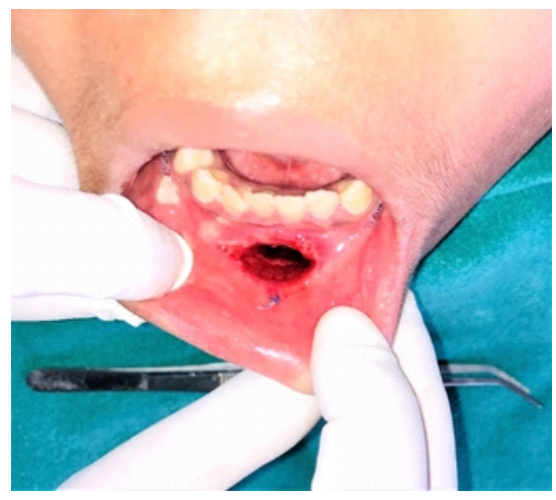

Figure 3. Marsupialization done under local anesthesia in the symphysis region

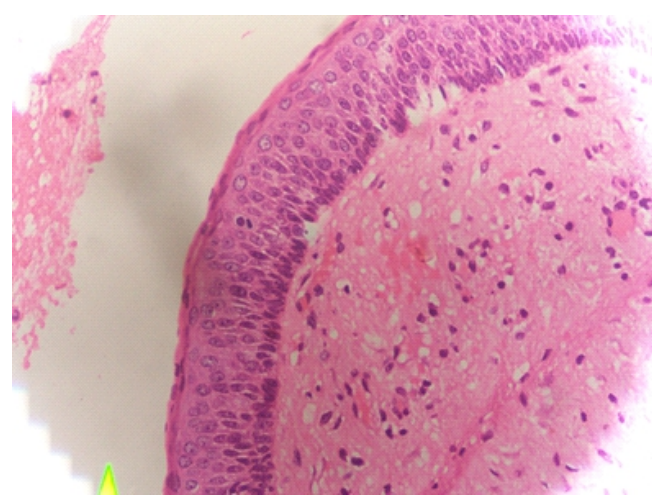

Figure 4. Histopathological section under $\mathbf{H}$ and $\mathbf{E}$ stain shows stratified squamous epithelium which is parakeratinized having 4-6 layers of thickness and basal cell layer is palisading arranged along with surface corrugation of the epithelium.

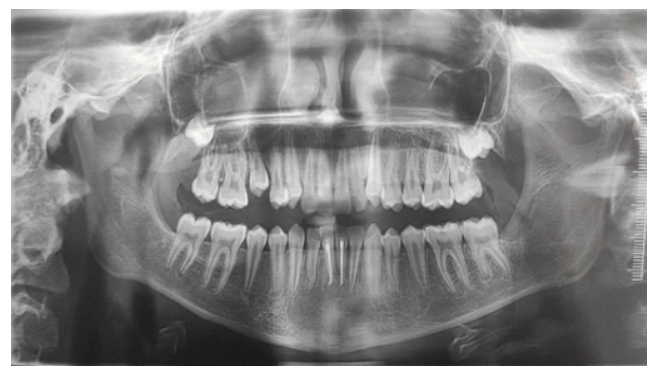

Figure 5. Post operative OPG after eight months of completion of treatment with healing cystic cavity

\section{DISCUSSION}

$\mathrm{OKC}$ is a common developmental odontogenic cyst and its biologic behavior is similar to a benign neoplasm. ${ }^{6} \mathrm{OKC}$ may be found in any age with peak prevalence between 20 to 40 years. ${ }^{7}$ The mandible is involved in 60 to $80 \%$ of cases with a marked tendency to occur in the posterior body and ascending ramus. ${ }^{6}$ Nevoid basal cell carcinoma syndrome (NBCCS) involves various skeletal, craniofacial, neurological, oropharyngeal, cutaneous, sexual, ophthalmic and cardiac anomalies. ${ }^{8}$

Multiple OKCs usually occur as a component of syndromes such as NBCCS, orofacial digital syndrome, Noonan syndrome, Ehler-Danlos syndrome and Simpson-GolabiBehmel syndrome. ${ }^{9}$ The present case showed only multiple OKCs without any other notable deformities such as basal cell carcinoma, skeletal defects, orofacial defects, stunted growth, bleeding diathesis, hyperextensible skin and hypermobile joints.

Therapeutic interventions of $\mathrm{OKCs}$ include resection, decompression and enucleation, combined with adjuvant cryotherapy with Carnoy's solution and peripheral ostectomy. ${ }^{10}$ Adjuvent therapies were addressed later after enucleation to minimize recurrence. Resection was already a treatment option, gained more prominence when OKC was classified as an odontogenic tumor. The reclassification of $\mathrm{OKC}$ as an odontogenic cyst stimulates the choice of conservative treatments and reinforces the continuity of investigations on decompression and complementary treatments.

Various studies have demonstrated decompression produces recurrences equivalent or even lower than those of direct enucleation. The recurrence after decompression was $15.4 \%$ which was similar to that after direct enucleation. Some articles reported a recurrence greater than $30 \%$ when decompressed OKCs did not undergo further enucleation, and this percentage might be related to the presence of residual cystic epithelium and possible satellite microcysts. ${ }^{11}$

The use of Carnoy's solution in combination with peripheral ostectomy may increase its safety margins and compensate for its deficiency when lesions are near soft tissues and between dental roots. Chow et al. demonstrated low recurrences $(4.3 \%$ and $0 \%$ respectively) when this combination of therapies was applied, but these authors did not specify the follow-up period. $^{12}$

OKCs related to NBCCS have more aggressive behavior and higher recurrence rates than non-syndromic ones. This characteristic is due to a high proliferation rate of the epithelial linings in the syndromic cases. ${ }^{13}$

\section{CONCLUSION}

In any patient with the presence of multiple OKCs, careful histopathologic examination, detailed family history and thorough clinical examination should be performed.In addition, chest and skull radiographs, panoramic radiographs 
of the jaw, MRI and CT scan should be advised. As shown in various studies, syndromic cases have a female predominance. Hence, suspected female patients should undergo pelvic ultrasonography. Moreover, a complete clinical examination and long-term follow up must be performed to detect any other features associated with NBCCS.

\section{CONFLICT OF INTEREST}

None

\section{REFERENCES}

1. Philipsen HP. On Keratocyst in the jaws. Tandlaegebladet. 1956;60:963-80.

2. Sholapurkar AA, MallelaVarun R, Pai KM. Non-syndromic multiple odontogenic keratocysts: Report of case. Revista de Clinica e Pesquisa Odontologica. 2008;4:193-9.

3. Sharif FN, Oliver R, Sweet C, Sharif MO. Interventions for the treatment of keratocystic odontogenic tumours. Cochrane Database of Systematic Reviews. 2010:9:1-12.

4. Philipsen HP. Keratocystic odontogenic tumor. In: Barnes L, Eveson JW, Reichart P, et al, editors. World Health Organization classification of tumours. Pathology and genetics of head and neck tumours. Lyon (France): IARC; 2005. p. 3067.

5. Regezi JA, Sciubba JJ, Jordan RC. Cysts of the jaws and neck. Oral pathology: clinical pathologic correlation, 6th edn. St. Louis: Saunders Elsevier. 2012;246-69.

6. Ahlfors E, Larsson Å, Sjögren S. The odontogenic keratocyst: a benign cystic tumor? Journal of Oral and Maxillofacial Surgery. 1984 Jan;42(1):10-9.

7. Brannon RB. The odontogenic keratocyst: A clinicopathologic study of 312 cases. Part I. Clinical features. Oral Surgery, Oral Medicine, Oral Pathology. 1976 Jul;42(1):54-72.

8. Auluck A, Suhas S, Pai KM. Multiple odontogenic keratocysts: report of a case. Journal of the Canadian Dental Association. 2006 Sep;72(7):651-6.

9. Lindeboom JA, Kroon FH, de Vires J, van den Akker HP. Multiple recurrent and de novo odontogenic keratocysts associated with oral-facial-digital syndrome. Oral Surgery, Oral Medicine, Oral Pathology, Oral Radiology, and Endodontology. 2003 Apr;95(4):458-62.

10. Habibi A, Saghravanian N, Habibi M, Mellati E, Habibi M. Keratocystic odontogenic tumor: a 10-year retrospective study of 83 cases in an Iranian population. Journal of Oral Science. 2007;49(3):229-35.

11. Chrcanovic BR, Gomez RS. Recurrence probability for keratocystic odontogenic tumors: an analysis of 6427 cases. Journal of Cranio-Maxillofacial Surgery. 2017 Feb $1 ; 45(2): 244-51$
12. Hsun-Tau C. Odontogenic keratocyst: a clinical experience in Singapore. Oral Surgery, Oral Medicine, Oral Pathology, Oral Radiology, and Endodontology. 1998 Nov;86(5):573-7.

13. Dominguez FV, Keszler A. Comparative study of keratocysts, associated and non associated with nevoid basal cell carcinoma syndrome. Journal of Oral Pathology \& Medicine.1988 Jan;17(1):39-42. 